\title{
The microbiome of Crohn's disease aphthous ulcers
}

\author{
Claire L. O'Brien ${ }^{1,2^{*}}$, Christopher J. Kiely ${ }^{1}$ and Paul Pavli ${ }^{1,2}$
}

\begin{abstract}
Background: Reduced intestinal microbial diversity and bacterial imbalance (dysbiosis) are seen in studies of Crohn's disease. As it is difficult to obtain biopsy samples before disease presentation, the earliest mucosal lesions in Crohn's disease, aphthous ulcers, present the best chance at assessing microbial communities at the onset of disease or a new flare. The aim of our study was to compare the microbial communities of aphthous ulcers and adjacent normal mucosa from patients with Crohn's disease with normal mucosa from controls.

Results: We did not observe bacterial imbalance or reduced alpha diversity in Crohn's disease aphthous ulcers and adjacent mucosa, relative to control biopsies. Bacteroides were common to all Crohn's disease and control samples, and there were no bacterial taxa unique to aphthous ulcers. The relative abundance of Faecalibacterium was not reduced in aphthous ulcers relative to control mucosa, and was not more likely to be detected in control samples.
\end{abstract}

Conclusions: In contrast to well-documented changes seen in late-stage Crohn's disease, microbial communities of aphthous ulcers do not display evidence of bacterial imbalance or reduced diversity. Our data suggest that dysbiosis occurs during active disease, and improves when patients are in remission.

Keywords: Crohn disease, Microbiome, Inflammatory bowel disease

\section{Background}

Crohn's disease (Crohn's disease), a chronic, relapsing inflammatory disease of the gastrointestinal tract, is thought to result from an aberrant, ongoing immune response to bacteria, in genetically susceptible individuals. Over 200 gene variants are associated with IBD, with just over 30 of these being Crohn's disease-specific [1]. Analysis of these gene variants suggests that hostmicrobe interactions are crucial in the development of Crohn's disease.

Several lines of evidence suggest that microbes play a role in either the onset or perpetuation of Crohn's disease. The earliest lesion in Crohn's disease is the aphthous ulcer, which overlies Peyer's patches in the small bowel, and lymphoid follicles in the large bowel. These lymphoid aggregates are the site of luminal antigen sampling by innate immune cells. Granulomas, which are a

\footnotetext{
*Correspondence: claire.obrien@anu.edu.au

${ }^{2}$ Gastroenterology and Hepatology Unit, Canberra Hospital, LVl 5, Bldg

10, Canberra Hospital, Yamba Drive, Garran, Canberra, ACT 2605, Australia

Full list of author information is available at the end of the article
}

histological hallmark of Crohn's disease, contain bacteria [2]. Temporary diversion of the fecal stream to a proximal ileostomy prevents the recurrence of inflammation in down-stream mucosal sites [3]. Numerous studies have shown that the gut microbiome is dysregulated in Crohn's disease, both in terms of its species composition and its function [4-6].

Reduced alpha diversity (mean number of bacterial species in a given sample) is frequently observed in the microbial communities of Crohn's disease mucosa when compared to mucosa from healthy controls, and cannot be attributed to inter-individual variation in the gut microbiome [7]. Studies consistently show that the gut microbiome of Crohn's disease patients is depauperate, in particular butyrate-producing Faecalibacterium prausnitzii and Roseburia [8-11]. Other groups of bacteria, such as Enterobacteriaceae, which includes Escherichia coli, are increased in Crohn's disease relative to controls $[4,12-14]$, and their abundance has been shown to correlate with disease severity [4]. Although a number of bacterial taxa have been implicated in Crohn's disease, no single causative organism has been identified. 
There is tremendous inter-individual variation in the gut microbiome of healthy individuals; however, despite this, the functional capacity of each individual's microbiome remains similar [7]. Conversely, modest differences in the taxonomic composition of the gut microbiome of patients with IBD are associated with major changes in its function [15]. These changes may reflect the response of bacteria to an inflamed gut, as enrichment in microbial pathways that enable bacteria to cope with oxidative stress, evade immune responses, and take up host metabolites without prior synthesis (auxotrophy) is observed. There are also corresponding reductions in short chain fatty acid (SCFA) and amino acid biosynthesis, as well as gut carbohydrate metabolism [15]. Proving that changes in the gut microbiome precede the onset of disease, cause a disease flare, or are a consequence of inflammation remains challenging.

It is almost impossible to obtain mucosal samples from people prior to the development of IBD, and only a few studies have assessed the mucosal microbiome of patients with Crohn's disease at the onset of disease. A large study of paediatric patients with new-onset Crohn's disease showed that species-richness was reduced in Crohn's disease, and that the abundance of several taxa was altered [4]. However, an inflammatory response was well established in $96 \%$ of the patients recruited to this study [mild (PCDAI 10-30)-moderate/severe disease (PCDAI $>30)$ ]. Dysbiosis is associated with other inflammatory conditions, such as obesity [16] and Type 2 diabetes [17], suggesting that chronic inflammation drives changes in the gut microbiome. A study by Kiely et al. showed that the mucosal microbiome of patients with inflammatory bowel disease (IBD) fluctuates over time, with greater changes observed in patients who had ongoing microscopic inflammation [18]. The majority of studies support the idea that dysbiosis is a common response to chronic inflammation.

The earliest mucosal lesions in Crohn's disease, aphthous ulcers, are small (1-5 $\mathrm{mm})$ superficial ulcerations surrounded by a ring of erythema then normal surrounding mucosa [19]. These lesions overlie the follicle associated epithelium (FAE) of the small bowel (Peyer's patches) and large bowel (lymphoid follicles) [20], can be found in $70 \%$ of patients with Crohn's disease [21], appear more commonly in the distal ileum $[19,21]$, and can develop into larger, transverse linear ulcers [19]. Approximately $10 \%$ of the epithelial cells of the FAE are microfold cells, commonly referred to as ' $M$ ' cells. These cells have a reduced glycocalyx and blunted microvilli, and are highly specialized in phagocytosis and transcytosis of luminal antigens, which they package in vesicles and deliver to underlying immune cells [22].
Numerous bacterial pathogens, including Yersinia pseudotuberculosis [23], Mycobacterium tuberculosis [24], Salmonella typhimurium [25], Shigella flexneri [26], and Escherichia coli $[27,28]$ exploit M cells to cross the epithelial barrier and cause infection. Adherent invasive E. coli (AIEC), which have been implicated in Crohn's disease, target $M$ cells on Peyer's patches through the expression of one of two major long polar fimbriae (lpfA) operons, which allows them to translocate the intestinal epithelial barrier [29]. However not all AIEC strains

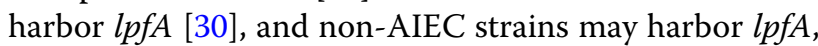
suggesting that this is not the only mechanism by which AIEC exploit M cells of the FAE.

Because of the difficulty in obtaining biopsy samples before Crohn's disease presentation, aphthous ulcers represent the earliest stage at which microbial communities can be assessed at the onset of disease or a disease flare [31]. The aim of our study was to compare the microbial communities of aphthous ulcers and adjacent mucosa from individuals with Crohn's disease with mucosa from healthy controls, to determine whether or not specific bacteria, or an imbalance in the gut microbiome, are present in the initial Crohn's disease lesion. This is the first study to assess the bacterial community composition of aphthous ulcers in Crohn's disease.

\section{Results}

\section{Subject characteristics}

The clinical characteristics of the 29 patients who underwent colonoscopy are summarised in Table 1. Two patients were on antibiotics at the time of the procedure (Patients 6 and 12 with Crohn's disease). The average time from diagnosis of IBD was 8.9 years (range $0-25$ years).

\section{Sequence coverage and diversity}

A total of 400,709 raw 16S rRNA gene sequences were generated from all samples, giving an average coverage of 9773 sequences per sample. The diversity of the microbial communities of Crohn's disease aphthous ulcers and adjacent normal mucosa, and healthy control mucosa, was estimated using the Shannon index. The samples from patients with Crohn's disease had similar diversity indices to the mucosa from healthy controls (ANOVA: $\mathrm{F}_{(1,27)}=0.0576, \mathrm{p}>\mathrm{F}=0.8125$, Fig. 1).

\section{Microbial community structure}

We did not observe bacterial imbalance in the majority of biopsies from Crohn's disease patients, including the aphthous ulcers, based on the relative abundance of the major phyla when compared to control samples (Fig. 2). An analysis of similarities (ANOSIM) revealed that the composition of the microbial communities of aphthous ulcers did not differ significantly from adjacent mucosa 
Table 1 Patient and sample characteristics

\begin{tabular}{|c|c|c|c|c|c|c|c|c|c|}
\hline Sample ID & Age (years) & Gender & Disease status & Biopsy location & $\begin{array}{l}\text { Years } \\
\text { since diagnosis }\end{array}$ & $\begin{array}{l}\text { Montreal } \\
\text { location }\end{array}$ & $\begin{array}{l}\text { Montreal } \\
\text { behaviour }\end{array}$ & Antibiotics & $\begin{array}{l}\text { Indication } \\
\text { for colonoscopy } \\
\text { (controls) }\end{array}$ \\
\hline ISBAU & 40 & M & $C D$ & TI ulcer & 8 & L1 & B1 & N & \\
\hline $1 \mathrm{SB}$ & & & & CM & & & & & \\
\hline $2 S B A U$ & 29 & $\mathrm{~F}$ & $C D$ & TI ulcer & 7 & L3 & B1 & N & \\
\hline $2 S B$ & & & & $\mathrm{Tl}$ & & & & & \\
\hline $3 \angle B A U$ & 37 & M & $C D$ & SIG ulcer & 18 & L2 & B1 & N & \\
\hline $3 \mathrm{LB}$ & & & & $A C$ & & & & & \\
\hline $4 S B A U$ & 32 & M & $C D$ & TI ulcer & 3 & L3 & B2p & N & \\
\hline $4 \mathrm{SB}$ & & & & $\mathrm{Tl}$ & & & & & \\
\hline $5 \mathrm{LBAU}$ & 30 & M & $C D$ & SIG ulcer & 6 & L3 & B1 & N & \\
\hline $5 \mathrm{LB}$ & & & & SIG & & & & & \\
\hline 6RECAU & 50 & $\mathrm{~F}$ & $C D$ & REC ulcer & 0 & L2 & B1 & Y & \\
\hline 6REC & & & & REC & & & & & \\
\hline 7LBAU & 40 & M & $U C$ & SIG ulcer & 25 & E3 & S2 & N & \\
\hline $7 \mathrm{LB}$ & & & & DC & & & & & \\
\hline 8SBAU & 34 & $\mathrm{~F}$ & $C D$ & CM ulcer & 15 & L3 & B2 & N & \\
\hline $8 \mathrm{SB}$ & & & & ICV & & & & & \\
\hline $9 S B A U$ & 22 & $\mathrm{~F}$ & $C D$ & CM ulcer & 0 & L3 & B1 & N & \\
\hline $9 S B$ & & & & CM & & & & & \\
\hline 10RECAU & 22 & $\mathrm{~F}$ & $C D$ & REC ulcer & 0 & L3 & B1 & N & \\
\hline 10REC & & & & REC & & & & & \\
\hline 11SBAU & 50 & M & $C D$ & TI ulcer & 10 & L3 & B3p & N & \\
\hline $11 \mathrm{SB}$ & & & $C D$ & $\mathrm{Tl}$ & & & & & \\
\hline $12 \mathrm{LBAU}$ & 28 & M & $C D$ & SIG ulcer & 15 & L3 & B2 & Y & \\
\hline $12 \mathrm{LB}$ & & & & DC & & & & & \\
\hline NB1 & 58 & M & NC & DC & - & & & & Rectal bleeding \\
\hline NB2 & 31 & $\mathrm{~F}$ & NC & $A C$ & - & & & & Constipation \\
\hline NB5 & 55 & & NC & $\mathrm{Tl}$ & - & & & & Altered bowel habit \\
\hline NB11 & 33 & M & NC & $\mathrm{Tl}$ & - & & & & Diarrhoea \\
\hline NB12 & 38 & M & NC & $\mathrm{Tl}$ & - & & & & Rectal bleeding \\
\hline NB13 & 35 & M & NC & REC & - & & & & Rectal bleeding \\
\hline NB15 & 57 & M & NC & $\mathrm{Tl}$ & - & & & & $\begin{array}{l}\text { Rectal bleeding, } \\
\text { abdominal pain }\end{array}$ \\
\hline NB17 & 43 & M & NC & $\mathrm{Tl}$ & - & & & & Rectal bleeding \\
\hline NB18 & 34 & M & NC & $\mathrm{Tl}$ & - & & & & $\begin{array}{l}\text { Rectal bleeding, } \\
\text { FHCRC }\end{array}$ \\
\hline NB21 & 53 & $\mathrm{~F}$ & NC & $\mathrm{Tl}$ & - & & & & $\begin{array}{l}\text { Bloating, abdominal } \\
\text { pain }\end{array}$ \\
\hline NB23 & 43 & M & NC & $\mathrm{Tl}$ & - & & & & FHCRC \\
\hline NB26 & 60 & $\mathrm{~F}$ & NC & SIG & - & & & & Rectal bleeding \\
\hline NB27 & 58 & M & NC & $\mathrm{Tl}$ & - & & & & Polyp surveillance \\
\hline NB29 & 37 & $\mathrm{~F}$ & NC & $\mathrm{Tl}$ & - & & & & Cancer surveillance \\
\hline NB37 & 48 & $\mathrm{~F}$ & NC & $\mathrm{Tl}$ & - & & & & $\begin{array}{l}\text { Fever of unknown } \\
\text { origin }\end{array}$ \\
\hline NB39 & 31 & $\mathrm{~F}$ & NC & $\mathrm{Tl}$ & - & & & & Abdominal pain \\
\hline NB48 & 20 & & NC & $A C$ & - & & & & Rectal bleeding \\
\hline
\end{tabular}

$C D$ Crohn's disease, UC ulcerative colitis, $N C$ normal control, $A U$ aphthous ulcer, $S B$ small bowel, $L B$ large bowel, $R E C$ rectum, $S / G$ sigmoid colon, $D C$ descending colon, $A C$ ascending colon, $I C V$ ileocaecal valve, $C M$ caecum, $T$ I terminal ileum, $N B$ normal (mucosa) biopsy, $F$ female, $M$ male, $L 1$ ileal CD, $L 2$ colonic $C D, L 3$ Ileocolonic CD, E3 pancolitis ulcerative colitis (proximal to splenic flexure), $B 1$ non-stricturing non-penetrating, $B 2$, structuring, $B 3$ penetrating, $p$ perianal disease, $N$ 'no', $Y$ 'yes' $F H C R C$, family history of colorectal cancer 


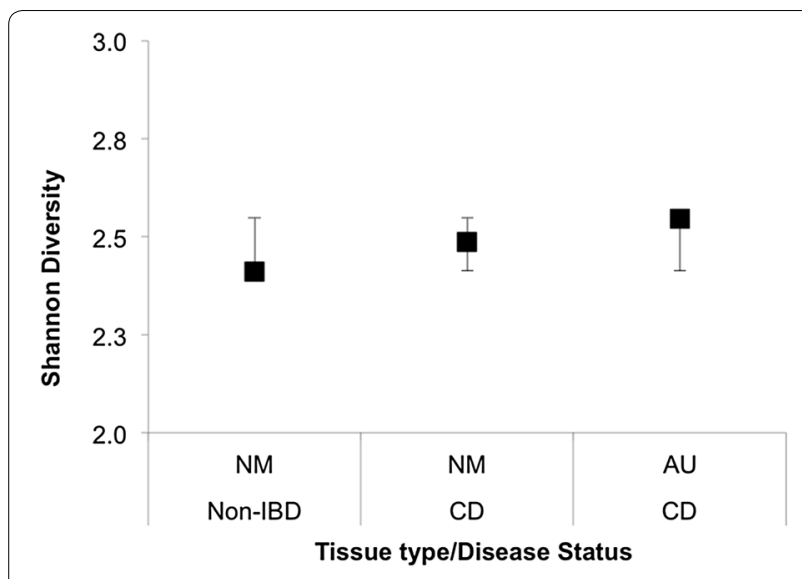

Fig. 1 Shannon diversity indices for the microbial communities of Crohn's disease aphthous ulcers and adjacent mucosa, and healthy control mucosa. Shannon Diversity Indices for the microbial communities of Crohn's disease aphthous ulcers and macroscopically normal adjacent mucosa, and healthy control (Non-IBD) mucosa. The means and $95 \% \mathrm{Cls}$ for each sample are depicted. There is no significant difference in the diversity of healthy control mucosa and Crohn's disease mucosa or aphthous ulcers (ANOVA: $\mathrm{F}_{(1,27)}=0.0576$, $\mathrm{p}>\mathrm{F}=0.8125)$. NM non-IBD normal mucosa from healthy controls without inflammatory bowel disease, NM CD adjacent normal mucosa from patients with Crohn's disease, $A \cup C D$ aphthous ulcers from patients with Crohn's disease

from the same patient $(\mathrm{p}=0.973)$, or from mucosa from healthy controls $(p=0.668)$. The average relative abundance of Firmicutes, which are often decreased in Crohn's disease [32], was: Crohn's disease mucosa, 67\%; Crohn's disease aphthous ulcers, 62\%; control mucosa, $47 \%$. The average relative abundance of Bacteroidetes was: Crohn's disease mucosa, 27\%; Crohn's disease aphthous ulcers, 33\%; control mucosa, $46 \%$. The average relative abundance of Proteobacteria, which are usually increased in Crohn's disease [32], was: Crohn's disease mucosa, 6\%; Crohn's disease aphthous ulcers, 5\%; control mucosa, 4\%. Control patient NB17 was excluded from the above analyses, because their microbiome comprised 99\% Pseudomonas, and was therefore a clear outlier.
A distance matrix was calculated for each sample using Oneway PERMANOVA and the Bray-Curtis similarity measure. The matrix was plotted in two dimensions using non-metric dimensional scaling (NMDS) (Fig. 3). The distance between two points in Fig. 3 is directly proportional to the Bray-Curtis similarity value for two samples, such that two samples that are close together have more similar microbial communities than those positioned further apart. The NMDS plot reveals that samples do not cluster by disease status. Samples from the same patient (aphthous ulcers and adjacent normal mucosa) are, on average, more similar to each other than samples from other patients. The distance between aphthous ulcer and adjacent mucosa samples from newly diagnosed patients (6REC/6RECAU; 9SB/9SBAU; 10REC/10RECAU) are similar to that of patients with established disease. Samples from patient 12 with Crohn's disease, who had been on antibiotics (12LB, 12LBAU), and NB17 (control) were removed from the analysis, as they were clear outliers. The same trends observed above, were also observed using the Jaccard and Theta YC algorithims, also using NMDS to plot the data.

A similarity percentage (Simper) analysis was conducted to determine the percent contribution of each bacterial family to the Bray-Curtis dissimilarity measure, for aphthous ulcers and mucosa from healthy controls (Table 2). Results are reported for families where the average dissimilarity was $>0.1 \%$. The following families had the highest average dissimilarity values: Bacteroidaceae (11.93\%); Lachnospiraceae (9.64\%); Ruminococcaceae (7.08\%); Prevotellaceae (5.08\%); Clostridiaceae_1 (2.36\%); and Erysipelotrichaceae (2.18\%). Of these, Lachnospiraceae, Clostridiaceae_1, Erysipelotrichaceae, and Enterococcaceae had a higher percentage contribution in aphthous ulcers compared to control mucosa.

\section{Presence/absence of bacterial taxa}

No bacterial taxa were unique to patients with Crohn's disease. Bacteroides was the only taxon common to all patients and controls, after those on antibiotics were

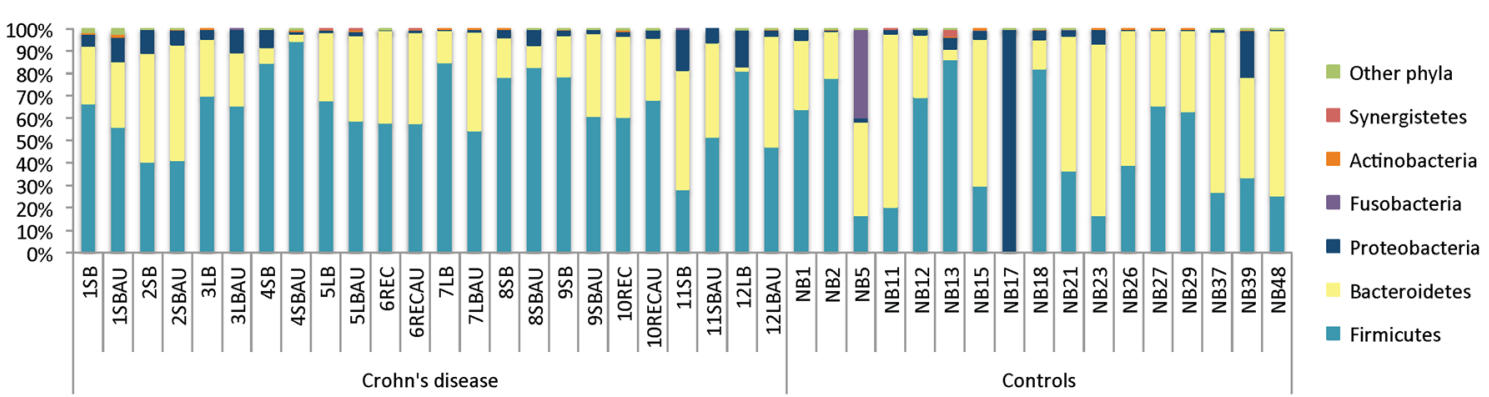

Fig. 2 Relative abundance of the dominant bacterial phyla for Crohn's disease and control tissues. Crohn's disease tissues: number corresponds to patient; SB small bowel, SBAU small bowl aphthous ulcer, $L B$ large bowel, $L B A U$, large bowel aphthous ulcer, $R E C$ rectum, $R E C A U$ rectal aphthous ulcer. Controls: $N B$ normal (mucosa) biopsy; number corresponds to patient 


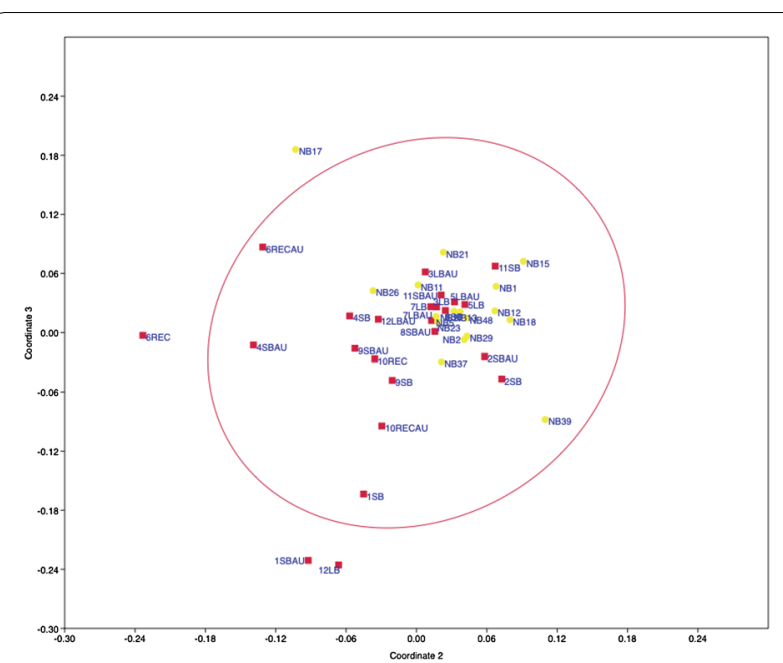

Fig. 3 Non-metric dimensional scaling (NMDS) plot of the microbial communities of Crohn's disease aphthous ulcers and adjacent mucosa, and healthy control mucosa. Non-metric dimensional scaling (NMDS) plot of the microbial 165 rRNA communities of Crohn's disease aphthous ulcers and adjacent macroscopically normal mucosa (red squares), and mucosa from healthy controls (yellow dots). The Bray-Curtis similarity index was used to determine the distance between two points; sites positioned close together share a greater fraction of their bacterial taxa than two samples further apart. Samples do not cluster according to disease status. Patient numbers precede the tissue type labels: $R E C$ rectum, $R E C A U$ rectal aphthous ulcer, $L B$ large bowel, $L B A U$ large bowel aphthous ulcer, SB small bowel, SBAU small bowel aphthous ulcer, NB normal (control mucosa) biopsy. NMDS stress $=0.40$

excluded (patients 6 and 12 with Crohn's disease). Clostridium cluster XIVa, and Lachnospiracea_incertae_sedis were present in the vast majority of Crohn's disease and control samples. The relative abundance of Faecalibacterium in Crohn's disease mucosa averaged $14 \%$ and was not significantly different to that of control mucosa, which averaged 10\% (ANOVA F $[1,25]=0.6869$, $\mathrm{p}>\mathrm{F}=00.415$ : Wilcoxon [Rank Sums] on untransformed data: $\left.\chi^{2}=0.394 \mathrm{p}>\chi^{2}=0.5302\right)$. There was no significant difference in the relative abundance of Faecalibacterium in Crohn's disease mucosa and aphthous ulcers (Match pairs $\mathrm{t}$ test $\left.\left[9_{\mathrm{DF}}\right]=0.957, \mathrm{p}>|\mathrm{t}|=0.363\right)$. Faecalibacterium was not significantly more likely to be detected in control samples: we detected Faecalibacterium in 94\% of normal controls and 91\% of patients with Crohn's disease $\left(\chi^{2}=0.101\right.$ $\mathrm{p}>\mathrm{X}^{2}=0.75$ ). Patients 6 and 12 with Crohn's disease, both on antibiotics, were excluded from these analyses.

\section{Discussion}

This is the first study to assess the microbiome of aphthous ulcers in Crohn's disease. Our study suggests that the microbiome is not imbalanced in the initial Crohn's disease lesion, relative to control mucosa. The alpha diversity, and composition of the microbiome of aphthous ulcers and adjacent mucosa from patients with IBD was similar to mucosa from controls. We found no evidence for a reduction in the genus, Faecalibacterium, which only contains one species, Faecalibacterium prausnitzii, and is commonly found to be decreased in Crohn's disease. We did not detect an increase in taxa that are usually over-represented in Crohn's disease mucosa, such as the family Enterobacteriaceae, which includes E. coli.

Bacterial community imbalance, or dysbiosis, is a common finding in IBD. Studies often report a decrease in protective groups (such as Lachnospiraceae, Roseburia, and Faecalibacterium), and a subsequent increase in pathobionts, (such as Proteobacteria, Ruminococcus, and Fusobacterium). Dysbiosis is likely to result from several factors. One study looked at the effects of inflammation, antibiotic exposure, and diet (exclusive enteral nutrition [EEN]) on the gut microbiome of paediatric patients with active Crohn's disease [33]. They found that each factor independently affected different bacterial taxa in the microbial community. They also showed that dysbiosis decreased with reduced intestinal inflammation, and that the microbiome of patients who responded to anti-TNF therapy and EEN became more similar to healthy controls than that of non-responders. These data support the idea that dysbiosis is a consequence, not cause, of inflammation. We did not control for diet in this study, however we did observe dysbiosis in patients who had consumed antibiotics. The degree or duration of inflammation in the aphthous ulcers may not have been great enough to affect the microenvironment, or to initiate dysbiosis.

A study by Lupp et al. [34]. showed that host-mediated inflammation in response to infection (Campylobacter jejuni) and oral administration of dextran sodium sulfate (DSS) leads to dysbiosis in a mouse model. In particular, they observed an expansion in Enterobacteriaceae. A study of the microbiome of a cohort of treatment-naïve new-onset patients with Crohn's disease, revealed that antibiotic use exaggerates dysbiosis [4]. They also showed that inflammatory conditions were strongly associated with a reduction in species richness and expansion of Enterobacteriaceae, as well as Bacteroidales, and Clostridiales.

The strength of this study was the ability to assess the microbiome of the initial Crohn's disease lesion (aphthous ulcer), before transmural inflammation and clinical manifestations developed for the first time, or for a new flare. Although our Crohn's disease cohort was small, we were able to demonstrate that dysbiosis is not a feature of aphthous ulcers. Similar sized cohorts of patients have demonstrated dysbiosis in samples obtained from patients with active Crohn's disease, including a reduction in Faecalibacterium [35, 36]. If 
Table 2 Similarity of percentages (SIMPER) results for bacterial community dissimilarity (Bray-Curtis) between bacterial families of aphthous ulcers and control mucosa

\begin{tabular}{|c|c|c|c|c|c|}
\hline Taxon (family) & Avg. Dissim \% & Contrib. \% & Cumulative \% & Mean aphthous & Mean control \\
\hline Bacteroidaceae & 11.93 & 24.54 & 24.54 & 0.31 & 0.36 \\
\hline Lachnospiraceae & 9.64 & 19.83 & 44.37 & 0.33 & 0.26 \\
\hline Ruminococcaceae & 7.08 & 14.57 & 58.93 & 0.13 & 0.15 \\
\hline Prevotellaceae & 5.08 & 10.45 & 69.39 & 0.02 & 0.09 \\
\hline Clostridiaceae_1 & 2.36 & 4.86 & 74.25 & 0.05 & 0.00 \\
\hline Erysipelotrichaceae & 2.18 & 4.49 & 78.74 & 0.04 & 0.03 \\
\hline Enterococcaceae & 1.97 & 4.04 & 82.78 & 0.04 & 0.00 \\
\hline Fusobacteriaceae & 1.25 & 2.58 & 85.36 & 0.00 & 0.03 \\
\hline Peptostreptococcaceae & 0.96 & 1.97 & 87.33 & 0.01 & 0.01 \\
\hline Sutterellaceae & 0.83 & 1.70 & 89.03 & 0.02 & 0.01 \\
\hline Enterobacteriaceae & 0.82 & 1.68 & 90.71 & 0.01 & 0.00 \\
\hline Streptococcaceae & 0.66 & 1.37 & 92.08 & 0.00 & 0.01 \\
\hline Comamonadaceae & 0.66 & 1.36 & 93.44 & 0.01 & 0.01 \\
\hline Veillonellaceae & 0.47 & 0.96 & 94.40 & 0.01 & 0.01 \\
\hline Porphyromonadaceae & 0.26 & 0.54 & 94.94 & 0.00 & 0.00 \\
\hline Hyphomicrobiaceae & 0.17 & 0.34 & 95.28 & 0.00 & 0.00 \\
\hline Clostridiales_Incertae_Sedis_XI & 0.17 & 0.34 & 95.62 & 0.00 & 0.00 \\
\hline Pasteurellaceae & 0.14 & 0.29 & 95.92 & 0.00 & 0.00 \\
\hline Synergistaceae & 0.12 & 0.25 & 96.16 & 0.00 & 0.00 \\
\hline Sphingomonadaceae & 0.11 & 0.22 & 96.39 & 0.00 & 0.00 \\
\hline Desulfovibrionaceae & 0.11 & 0.22 & 96.61 & 0.00 & 0.00 \\
\hline Rikenellaceae & 0.11 & 0.22 & 96.84 & 0.00 & 0.00 \\
\hline Flavobacteriaceae & 0.10 & 0.21 & 97.05 & 0.00 & 0.00 \\
\hline
\end{tabular}

Similarity of percentages (SIMPER) analysis showing the average dissimilarity (\%[Avg. Dissim]) of bacterial families represented in aphthous ulcers and control mucosa. Percentage contribution (Contrib. \%) is the mean contribution divided by the mean dissimilarity across samples. Only Families with an average dissimilarity $>0.10 \%$ are shown

dysbiosis were a feature of the aphthous ulcer microbiome, we would likely have observed it in a number of our Crohn's disease patients.

It is unclear when dysbiosis of the gut microbiome develops in patients with Crohn's disease, and if it becomes progressively worse with each disease flare. One study assessed the gut microbiome of unaffected genetically-linked relatives of children with Crohn's disease. The unaffected relatives had alterations in their gut microbiome in the direction of their relatives with Crohn's disease, but did not display a distinct dysbiosis [36]. The findings of this study suggest that dysbiosis develops close to disease onset, or as a consequence of the disease process. There is some evidence to suggest that dysbiosis improves over time, but is still evident, in patients with complete mucosal healing or who have responded to treatment [35]. Only one patient with Crohn's disease in this study (CD11) had previous surgery, all other patients were in remission, or had only mild symptoms. Patients with a long history of mild disease may be less likely to have gut microbiome imbalance.
If dysbiosis does improve in the absence of active disease, then interventions aimed at restoring the gut microbiome may be effective in increasing gut microbial diversity. Reduced alpha diversity could lead to a breakdown in the functional redundancy of gut communities, which may exacerbate symptoms. It would be important to administer interventions, such as pro-, pre- and syn-biotics, in the absence of inflammation, as attempts to establish or nourish bacteria that do not cope well in an environment of chronic inflammation and oxidative stress may be futile.

\section{Conclusions}

Our data suggest that dysbiosis is a consequence of the inflammatory disease process, as it was not observed in the initial lesion. We did not detect dysbiosis in the three patients who were newly diagnosed at the time of sampling, nor in patients with more established disease. Longitudinal studies aimed at assessing the gut microbiome before disease onset and throughout successive flares would provide further insight into the nature and 
development of dysbiosis, but acquiring samples from patients prior to diagnosis remains problematic.

\section{Methods}

\section{Patient and sample characteristics}

Biopsies were collected at the time of colonoscopy by a gastroenterologist, and all diagnoses of IBD were made based on standard criteria: clinical presentation and endoscopic/clinical findings. A total of 41 mucosal biopsies were used for the study: aphthous ulcers $(n=12)$ and adjacent normal mucosal biopsies $(\mathrm{n}=12)$ from patients with IBD, and normal mucosal biopsies from healthy controls $(n=17)$. Biopsies from healthy controls were selected from a larger pool, so that they resembled the biopsies from IBD patients with respect to gut region, age, and gender. All samples were stored in RNAlater ${ }^{\circledR}$ at $4{ }^{\circ} \mathrm{C}$ for $24 \mathrm{~h}$, then $-80^{\circ} \mathrm{C}$ until required. Table 1 outlines the characteristics for the study participants, including disease status and behaviour, gut location of biopsies, age, gender, and antibiotic usage. Only one patient (CD11) had prior surgery.

\section{DNA extraction and amplification}

Biopsies in RNAlater ${ }^{\circledR}$ were thawed and DNA was extracted using Qiagen DNeasy Blood and Tissue kits, with the addition of the enzymatic lysis buffer, beadbeating ( $5000 \mathrm{rpm} / 30 \mathrm{revs} / \mathrm{s}$ for $3 \mathrm{~min}$ using a Qiagen TissueLyser II), and RNase A steps, as described in the manufacturer's protocol. Extraction negative controls were included and were always negative. DNA was amplified using barcoded universal bacterial primers targeting the V1-V3 region of the $16 \mathrm{~S}$ rRNA gene, and PCR conditions, as previously described [5]. Both positive and negative controls were used for each PCR. DNA was quantified and quality-checked using an Agilent 2100 Bioanalyzer with DNA 1000 chips. Equimolar amounts of the PCR products were combined to make a $500 \mathrm{ng}$ library, which was used as template in the emulsion PCR prior to sequencing on a 454 Genome Sequencer FLXTitanium system. The sequencing was performed at the Biological Research Facility, ANU, Australia, according to the manufacturer's instructions (454 Life Sciences, Branford, Connecticut, USA). Signal processing and base calling were performed using 454 Sequencing Software V.2.6 (Roche).

\section{Sequence processing}

Sequence curation and processing were performed in Mothur [37] (v 1.32.1) as previously described [5]. Briefly, sequences were assessed for quality, trimmed of adaptors and barcodes (barcode mismatches allowed, 1 bp; primer mismatches, $2 \mathrm{bp}$ ), and chimeras removed using the Uchime code [38]. Sequences were aligned using the Silva database, and taxonomic assignments using the RDP 2012 training sets.

\section{Statistical analysis}

PAST3 was used to generate: non-metric dimensional scaling (NMDS) plots, using the Bray-Curtis similarity measure, using normalized family level taxonomic data as an input; one-way ANOSIM; one-way ANOVA; Simper analysis using the Bray-Curtis measure of similarity. JMP (v.9) was used to conduct one-way ANOVA of Shannon indices between Crohn's disease and control samples, and to conduct matched pairs $t$ test statistics of mean relative abundance figures for individual taxa.

\section{Authors' contributions \\ $C O$ 'B and PP designed the experiment. $\mathrm{CO}^{\prime} \mathrm{B}$ conducted the experiments and bioinformatics analyses, and wrote the paper. PP, CK critically reviewed the manuscript and conducted analyses. All authors read and approved the final manuscript.}

\section{Author details}

${ }^{1}$ Medical School, Australian National University, Canberra, ACT 2600, Australia. ${ }^{2}$ Gastroenterology and Hepatology Unit, Canberra Hospital, Lvl 5, Bldg 10, Canberra Hospital, Yamba Drive, Garran, Canberra, ACT 2605, Australia.

\section{Acknowledgements}

We thank Kerong Zhang of the Biological Research Facility at the Australian National University for his technical assistance, as well as the nurses who assisted with sample collection. Special thanks go to the study participants who allowed us to perform these studies.

\section{Competing interests}

The authors declare that they have no competing interests.

\section{Availability of data and materials}

Not applicable.

\section{Consent for publication}

Not applicable.

\section{Ethics approval and consent to participate}

Written informed consent was obtained from all subjects, and the study was approved by the ACT Health Human Research Ethics Committee (Protocol: ETH.5.07.464), and Australian National University Human Ethics Committee (Protocol: 2012/596)

\section{Funding}

This work was supported by a National Health and Medical Research Council Early Career Fellowship (CO'B, APP1091221).

\section{Publisher's Note}

Springer Nature remains neutral with regard to jurisdictional claims in published maps and institutional affiliations.

Received: 31 May 2018 Accepted: 27 Auqust 2018

Published online: 10 October 2018

References

1. Jostins L, Ripke S, Weersma RK, et al. Host-microbe interactions have shaped the genetic architecture of inflammatory bowel disease. Nature. 2012;491(7422):119-24. https://doi.org/10.1038/nature11582. 
2. Ryan P, Kelly RG, Lee G, et al. Bacterial DNA within granulomas of patients with Crohn's disease-detection by laser capture microdissection and PCR. Am J Gastroenterol. 2004;99(8):1539-43. https://doi.org/10.111 1/j.1572-0241.2004.40103.x.

3. Rutgeerts P, Goboes K, Peeters M, et al. Effect of faecal stream diversion on recurrence of Crohn's disease in the neoterminal ileum. Lancet. 1991:338(8770):771-4.

4. Gevers D, Kugathasan S, Denson LA, et al. The treatment-naive microbiome in new-onset Crohn's disease. Cell Host Microbe. 2014;15(3):382-92. https://doi.org/10.1016/j.chom.2014.02.005.

5. O'Brien CL, Pavli P, Gordon DM, et al. Detection of bacterial DNA in lymph nodes of Crohn's disease patients using high throughput sequencing. Gut. 2014;63(10):1596-606. https://doi.org/10.1136/gutjnl-2013-305320.

6. Kostic AD, Xavier RJ, Gevers D. The microbiome in inflammatory bowel disease: current status and the future ahead. Gastroenterology. 2014;146(6):1489-99. https://doi.org/10.1053/j.gastro.2014.02.009.

7. Human Microbiome Project C. Structure, function and diversity of the healthy human microbiome. Nature. 2012;486(7402):207-14. https://doi. org/10.1038/nature11234.

8. Vidal R, Ginard D, Khorrami $\mathrm{S}$, et al. Crohn associated microbial communities associated to colonic mucosal biopsies in patients of the western Mediterranean. Syst Appl Microbiol. 2015;38(6):442-52. https://doi. org/10.1016/j.syapm.2015.06.008.

9. Fujimoto T, Imaeda $\mathrm{H}$, Takahashi K, et al. Decreased abundance of Faecalibacterium prausnitzii in the gut microbiota of Crohn's disease. J Gastroenterol Hepatol. 2013;28(4):613-9. https://doi.org/10.1111/jgh.12073.

10. Hansen R, Russell RK, Reiff C, et al. Microbiota of de-novo pediatric IBD: increased Faecalibacterium prausnitzii and reduced bacterial diversity in Crohn's but not in ulcerative colitis. Am J Gastroenterol. 2012;107(12):1913-22. https://doi.org/10.1038/ajg.2012.335.

11. Chen L, Wang W, Zhou R, et al. Characteristics of fecal and mucosa-associated microbiota in Chinese patients with inflammatory bowel disease. Medicine. 2014;93(8):e51. https://doi.org/10.1097/MD.000000000000005 1.

12. Willing B, Halfvarson J, Dicksved J, et al. Twin studies reveal specific imbalances in the mucosa-associated microbiota of patients with ileal Crohn's disease. Inflam Bowel Dis. 2009;15(5):653-60. https://doi.org/10.1002/ ibd.20783.

13. Baumgart M, Dogan B, Rishniw M, et al. Culture independent analysis of ileal mucosa reveals a selective increase in invasive Escherichia coli of novel phylogeny relative to depletion of Clostridiales in Crohn's disease involving the ileum. ISME J. 2007;1(5):403-18. https://doi.org/10.1038/ ismej.2007.52.

14. Gophna U, Sommerfeld K, Gophna S, et al. Differences between tissueassociated intestinal microfloras of patients with Crohn's disease and ulcerative colitis. J Clin Microbiol. 2006;44(11):4136-41. https://doi. org/10.1128/JCM.01004-06

15. Morgan XC, Tickle TL, Sokol H, et al. Dysfunction of the intestinal microbiome in inflammatory bowel disease and treatment. Genome Biol. 2012;13(9):R79. https://doi.org/10.1186/gb-2012-13-9-r79.

16. Torres-Fuentes C, Schellekens H, Dinan TG, et al. The microbiota-gutbrain axis in obesity. Lancet Gastroenterol Hepatol. 2017. https://doi. org/10.1016/S2468-1253(17)30147-4.

17. Qin J, Li Y, Cai Z, et al. A metagenome-wide association study of gut microbiota in type 2 diabetes. Nature. 2012;490(7418):55-60. https://doi. org/10.1038/nature11450.

18. Kiely CJOB, Pavli P. Inflammation and changes in the inflammatory bowel disease microbiome over time. J Gastroenterol Hepatol. 2017;32(S2):118. https://doi.org/10.1111/jgh.13894.

19. Rutgeerts P, Geboes K, Vantrappen G, et al. Natural history of recurrent Crohn's disease at the ileocolonic anastomosis after curative surgery. Gut. 1984;25(6):665-72.
20. Morson BC. The early histological lesion of Crohn's disease. Proc R Soc Med. 1972;65(1):71-2.

21. Rickert RR, Carter HW. The "early" ulcerative lesion of Crohn's disease: correlative light- and scanning electron-microscopic studies. J Clin Gastroenterol. 1980;2(1):11-9.

22. Mabbott NA, Donaldson DS, Ohno H, et al. Microfold (M) cells: important immunosurveillance posts in the intestinal epithelium. Mucosal Immunol. 2013;6(4):666-77. https://doi.org/10.1038/mi.2013.30.

23. Marra A, Isberg RR. Invasin-dependent and invasin-independent pathways for translocation of Yersinia pseudotuberculosis across the Peyer's patch intestinal epithelium. Infect Immun. 1997;65(8):3412-21.

24. Owen RL. Uptake and transport of intestinal macromolecules and microorganisms by M cells in Peyer's patches-a personal and historical perspective. Sem Immunol. 1999;11(3):157-63. https://doi.org/10.1006/ smim.1999.0171.

25. Jones BD, Ghori N, Falkow S. Salmonella typhimurium initiates murine infection by penetrating and destroying the specialized epithelial $\mathrm{M}$ cells of the Peyer's patches. J Exp Med. 1994;180(1):15-23.

26. Sansonetti PJ, Arondel J, Cantey JR, et al. Infection of rabbit Peyer's patches by Shigella flexneri: effect of adhesive or invasive bacterial phenotypes on follicle-associated epithelium. Infect Immun. 1996;64(7):2752-64.

27. Gebert A, Rothkotter HJ, Pabst R. M cells in Peyer's patches of the intestine. Int Rev Cytol. 1996;167:91-159.

28. Siebers A, Finlay BB. M cells and the pathogenesis of mucosal and systemic infections. Trends Microbiol. 1996;4(1):22-9.

29. Chassaing B, Darfeuille-Michaud A. The interaction of Crohn's diseaseassociated Escherichia coli to Peyer's patches of the intestinal mucosa involves long polar fimbriae. Med Sci. 2011;27(6-7):572-3. https://doi. org/10.1051/medsci/2011276003.

30. O'Brien CL, Bringer MA, Holt KE, et al. Comparative genomics of Crohn's disease-associated adherent-invasive Escherichia coli. Gut. 2016. https:// doi.org/10.1136/gutjnl-2015-311059.

31. Rutgeerts PJ. From aphthous ulcer to full-blown Crohn's disease. Dig Dis. 2011;29(2):211-4. https://doi.org/10.1159/000323922.

32. Wright EK, Kamm MA, Teo SM, et al. Recent advances in characterizing the gastrointestinal microbiome in Crohn's disease: a systematic review. Inflam Bowel Dis. 2015;21(6):1219-28. https://doi.org/10.1097/MIB.00000 00000000382.

33. Lewis JD, Chen EZ, Baldassano RN, et al. Inflammation, antibiotics, and diet as environmental stressors of the gut microbiome in pediatric Crohn's disease. Cell Host Microbe. 2015;18(4):489-500. https://doi. org/10.1016/j.chom.2015.09.008.

34. Lupp C, Robertson ML, Wickham ME, et al. Host-mediated inflammation disrupts the intestinal microbiota and promotes the overgrowth of Enterobacteriaceae. Cell Host Microbe. 2007;2(3):204.

35. Shaw KA, Bertha M, Hofmekler T, et al. Dysbiosis, inflammation, and response to treatment: a longitudinal study of pediatric subjects with newly diagnosed inflammatory bowel disease. Genome Med. 2016;8(1):75. https://doi.org/10.1186/s13073-016-0331-y.

36. Ijaz UZ, Quince C, Hanske L, et al. The distinct features of microbial 'dysbiosis' of Crohn's disease do not occur to the same extent in their unaffected, genetically-linked kindred. PloS one. 2017;12(2):e0172605. https://doi.org/10.1371/journal.pone.0172605.

37. Schloss PD, Westcott SL, Ryabin T, et al. Introducing mothur: open-source, platform-independent, community-supported software for describing and comparing microbial communities. Appl Environ Microbiol. 2009;75(23):7537-41. https://doi.org/10.1128/AEM.01541-09.

38. Edgar RC, Haas BJ, Clemente JC, et al. UCHIME improves sensitivity and speed of chimera detection. Bioinformatics. 2011;27(16):2194-200. https ://doi.org/10.1093/bioinformatics/btr381. 